\title{
FLORISTIC COMPOSITION OF THE CERRADO IN THE PÉ-DE-GIGANTE RESER- VE (SANTA RITA DO PASSA QUATRO, SOUTHEASTERN BRAZIL)
}

\author{
Marco Antônio Batalha ${ }^{1}$ \\ Waldir Mantovani ${ }^{2}$
}

Recebido em 04/02/00. Aceito em 25/07/01.

\begin{abstract}
RESUMO - (Composição florística do cerrado na Reserva Pé-de-Gigante (Santa Rita do Passa Quatro, SP)). Estudamos uma área de 1225 ha, composta principalmente por cerrado, situada em Santa Rita do Passa Quatro, estado de São Paulo (21 $\left.1^{\circ} 36-38^{\prime} \mathrm{S}, 47^{\circ} 36-39^{\prime} \mathrm{W}\right)$. Em três fisionomias de cerrado (campo cerrado, cerrado sensu stricto e cerradão), coletamos, durante 18 meses, em excursões mensais, espécimes em fase fértil e os identificamos em nível específico. Encontramos 360 espécies, pertencentes a 236 gêneros e 69 famílias. As famílias mais ricas foram: Fabaceae, Asteraceae, Poaceae e Rubiaceae. As fisionomias savânicas foram mais ricas do que a florestal. A razão entre espécies arbustivo-arbóreas e herbáceo-subarbustivas foi de aproximadamente 2:1. Analisamos a flora como um todo e seus componentes herbáceo-subarbustivo e arbustivo-arbóreo separadamente, comparando-os com outras áreas disjuntas de cerrado. Dessa comparação, obtivemos valores de similaridade (índice de Sørensen) de 0,47 a 0,81, que mostraram que a diversidade 3 foi maior no componente herbáceosubarbustivo do que no componente arbustivo-arbóreo.
\end{abstract}

Palavras-chave - cerrado, savana, fisionomia, florística.

ABSTRACT - (Floristic composition of the cerrado in the Pé-de-Gigante Reserve (Santa Rita do Passa Quatro, southeastern Brazil)). We studied a 1225 ha area, composed mainly of cerrado, in Santa Rita do Passa Quatro,

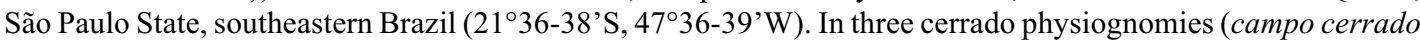
- a wooded savanna, cerrado sensu stricto - a woodland, and cerradão - a tall woodland), we collected all vascular plants in reproductive stage, and identified them to species level. We found 360 species, representing 236 genera and 69 families. The richest families were: Asteraceae, Fabaceae, Poaceae, and Rubiaceae. The savanna physiognomies were richer than the forest one. The ratio between herbaceous and woody species was approximately $2: 1$. We analysed the whole flora and its two components separately, woody and herbaceous, comparing them with other disjunct cerrado areas. We obtained similarity values (Sørensen index) from 0.47 to 0.81 , which showed that the 3 diversity of the cerrado was higher in the herbaceous component than in the woody one.

Key words - cerrado, savanna, physiognomy, floristics, woody: herbaceous ratio

\footnotetext{
${ }^{1,2}$ Depto. de Ecologia, Instituto de Biociências, Universidade de São Paulo, Caixa Postal 11.461, 05422-970, São Paulo, SP, Brasil. ${ }^{1}$ Pós-Graduação em Ecologia (marcobat@uol.com.br).

${ }^{2}$ Prof. Titular (wmantova@usp.br).
} 


\section{Introduction}

About $23 \%$ of the Brazilian territory (2 millions $\mathrm{km}^{2}$ ) were originally covered by cerrado vegetation (Ratter et al. 1992). Its core area covers the Brazilian Central Highlands, in the states of Minas Gerais, Mato Grosso, Mato Grosso do Sul, Goiás, Tocantins, Maranhão, and Piauí (Mantovani \& Martins 1993). Outlying cerrado areas also occur in other states, as in São Paulo (Ratter et al. 1992).

The cerrado vegetation is characterized by its wide physiognomic variation. According to Coutinho (1978), the cerrado goes from campo limpo, a grassland, to cerradão, a woodland. The intermediate physiognomies (campo sujo - a shrub savanna, campo cerrado - a wooded savanna, and cerrado sensu stricto - a woodland) are considered ecotones.

Accompanying the physiognomic variation, the cerrado vegetation presents a high floristic richness. Castro et al. (1999) compiled many floristic and phytosociological surveys carried out in cerrado areas and estimated the number of vascular plant species in this vegetation type ranging from 3,000 to 7,000. Mendonça et al. (1998) listed 6,429 species for the whole domain, including, besides the cerrado itself, other associated vegetation types, such as gallery forest, deciduous and semideciduous forest, and floodplain grassland.

The cerrado vegetation has two distinct floras, a woody one and an herbaceous one, which are antagonistic, because both are sun-loving (Coutinho 1978). The importance of the herbaceous flora gradually decreases from the campo limpo to the cerradão, while the importance of the woody flora increases in this direction (Coutinho 1978). Mantovani \& Martins (1993) compared some cerrado areas, and they found a species ratio of $1: 2$ to $1: 3$ between the woody and the herbaceous floras. Castro et al. (1999) emphasized the almost complete absence of studies that sampled the herbaceous component, in spite of its high richness.
Our aim was to carry out a floristic survey in a disjunct cerrado area, distinguishing its physiognomies. This survey intends to increase the knowledge of the cerrado flora, especially of the frequently neglected herbaceous component, and to contribute to phytogeographical studies.

\section{Material and methods}

The Pé-de-Gigante Reserve is located in Santa Rita do Passa Quatro Municipality, São Paulo State, southeastern Brazil, between 21 36-38'S and 47 36-39'W, under a Cwag' (Köppen 1948) or $\mathrm{B}_{3} \mathrm{~B}_{3}{ }_{3} \mathrm{~W}$ (Thornthwaite \& Mather 1955) climate type, at 590 to $740 \mathrm{~m}$ high, on Red-Yellow Latosol (Pivello et al. 1998). Its name ("Pé-de-Gigante" or "Giant's foot") was given due to the presence of a foot-shaped geomorphological formation in the Paulicéia Stream drainage basin (Fig. 1). The study area covers $1225 \mathrm{ha}$, in which are found mainly cerrado physiognomies: campo sujo ( $0.25 \%$ of the total area), campo cerrado $(7.9 \%)$, cerrado sensu stricto $(79.0 \%)$ and cerradão (11.1\%). Other vegetation types are also present, such as floodplain grassland, gallery forest and seasonal semideciduous forest. A more detailed characterization of the study area can be found in Pivello et al. (1998, 1999a).

First, the reserve was mapped with remote sensing techniques, using a vegetation index that measures the green biomass, with which the occurrence of cerrado regions was assigned (Bitencourt et al. 1997, Pivello et al. 1999a). We distinguished the cerrado physiognomies following the "forest-ecotone-grassland" concept (Coutinho 1978). Then, in each cerrado physiognomy, except the campo sujo due to its small extension, we carried out floristic surveys in monthly field trips, each one with $23 \mathrm{hr}$ sampling effort, from September 1995 to February 1997, when we collected all vascular plant species in reproductive phase. We lodgeal the collected exsicata in the herbarium of São Paulo Botanical Institute (SP). P l a n t s were classified in families according to the sys- 
tem proposed by Judd et al. (1999). The species were classified in life forms in accordance with Raunkiaer's system, adapted by Mueller-Dombois \& Ellenberg (1974). We considered the phanerophyte species as belonging to the woody component and the non-phanerophytes species as belonging to the herbaceous one. We calculated the floristic similarity among the different physiognomies at species level, grouping them in clusters, using average-linking as agglomerative algorithm
(Jongman et al. 1995) and Sørensen index as distance measure (Magurran 1988). The results were compared in the same way with other floristic surveys, in which similar methods were used (Mantovani \& Martins 1993, Batalha et al. 1997).

\section{Results and Discussion}

In the cerrado physiognomies (campo cerrado, cerrado sensu stricto, and cerradão), 360

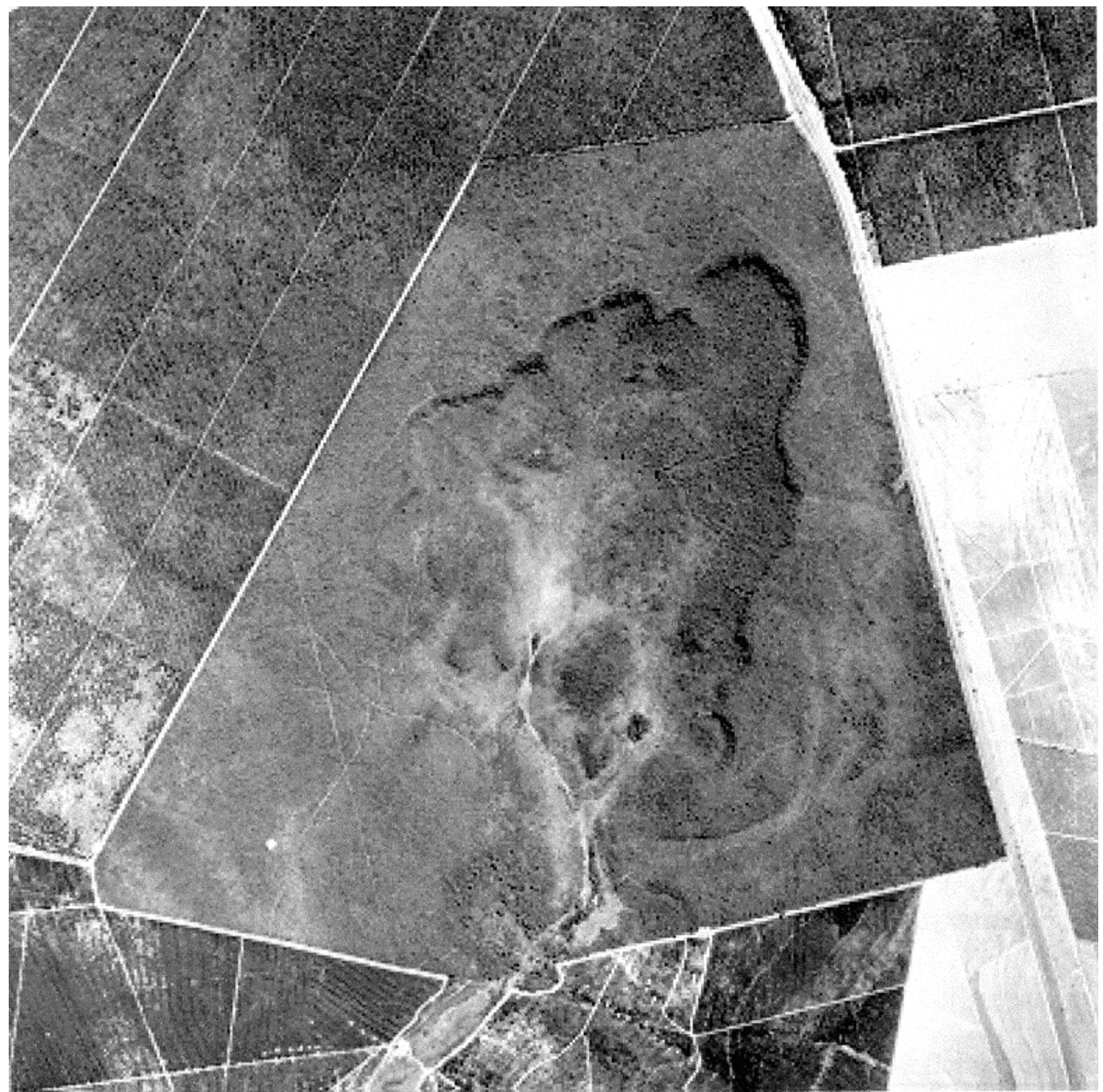

Figure 1. Aerial photograph (1:40.000) of the Pé-de-Gigante Reserve, Santa Rita do Passa Quatro, São Paulo State, Brazil (21³6-38’S, 47³6-39’W). 
species were found, belonging to 236 genera and 69 families (Tab. 1 and 2). The richest families were: Fabaceae, Asteraceae, Poaceae, Rubiaceae, Bignoniaceae, Myrtaceae, Malpighiaceae, Malvaceae, and Apocynaceae, together accounting for $57.8 \%$ of collected species (Fig. 2).

These families are also the most representative ones in other cerrado areas, such as Lagoa Santa (Warming 1892), Triângulo Mineiro (Goodland 1979), Brasília (Ratter 1980), Moji Guaçu (Mantovani \& Martins 1993), and Pirassununga (Batalha et al. 1997).

Of the total collected species, 16 (4.4\%) are weeds that does not occur spontaneously in cerrado sites (Mendonça et al. 1998). In this vegetation type, this is mainly a consequence of the fragmentation and edge effect (Pivello et al. 1998, 1999b). Mendonça et al. (1998) listed for the whole Cerrado Domain 289 weedy species, or $4.5 \%$ of its total flora, the same proportion observed in the Pé-de-Gigante flora. Plant invasions are a major problem in cerrado fragments, occuring pratically in every one of them, dominating in patches and outcompeting native species (Pivello et al. 1999b). This situation occurs also in the Pé-de-Gigante Reserve, where African grasses, especially Melinis minutiflora P. Beauv. and Brachiaria decumbens Stapf, are spreading fast (Pivello et al. 1999c).

A total of 272 species were found in the campo cerrado; 308 , in the cerrado sensu stricto; and 148, in the cerradão (Tab. 2). Following the "forest-ecotone-grassland" concept (Coutinho 1978), the ecotonal physiognomies are expected to be richer, because they contain species from both the herbaceous and the woody components. Indeed, the existing ecotonal physiognomies in the Pé-de-Gigante Reserve, campo cerrado and cerrado sensu stricto, were richer than one of the extremes, cerradão. The woody to herbaceous species ratio for all three physiognomies together (cerrado sensu lato) was approximately 1:2 (Tab. 2). This ratio increased from cerradão, in which the number of woody species was higher than those of herbaceous species, to campo cerrado (Tab. 2). Once again, this is expected according to the "forestecotone-grassland" concept (Coutinho 1978),

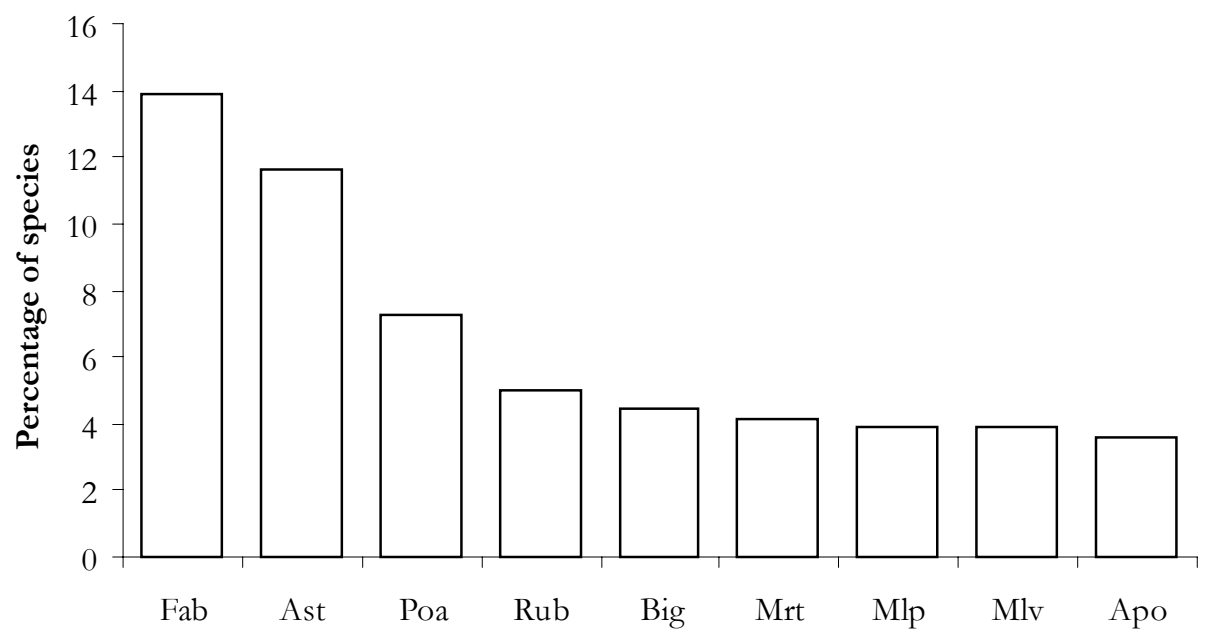

Figure 2. Percentage of species in the richest families in cerrado physiognomies in the Pé-de-Gigante Reserve, Santa Rita do Passa Quatro, São Paulo State, Brazil (21³6-38’S, 47³6-39’ W). Key: Fab = Fabaceae, Ast $=$ Asteraceae, Poa $=$ Poaceae, Rub = Rubiaceae, Big = Bignoniaceae, Mrt = Myrtaceae, Mlp = Malpighiaceae, Mlv = Malvaceae, and Apo = Apocynaceae. Other families accounted for $42.2 \%$ of the total number of species. 


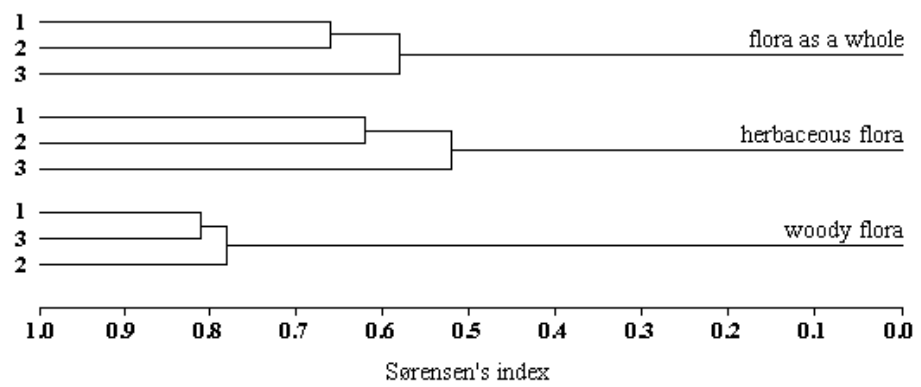

Figure 3. Cluster analysis of cerrado floras. 1 = Pé-de-Gigante Reserve, Santa Rita do Passa Quatro, São Paulo State,

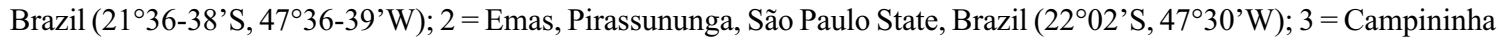
Farm, Moji Guaçu, São Paulo State, Brazil (22¹5-16’S, 4708-12’W).

which predicts a gradual decrease in the number of woody species from cerradão to campo limpo. In Brasília (Ratter 1980), this ratio was also 1:2, while in Lagoa Santa (Warming 1892) and Moji Guaçu (Mantovani \& Martins 1993) it was 1:3. In Santa Rita do Passa Quatro and Brasília, there is a predominance of closed cerrado physiognomies (cerrado sensu stricto and cerradão). In Lagoa Santa and Moji Guaçu, on the other hand, open cerrado physiognomies (campo sujo and campo cerrado) prevail, explaining the higher proportion of herbaceous species in these two areas.

In the three cerrado physiognomies sampled in the Pé-de-Gigante Reserve, the highest similarity was between campo cerrado and cerrado sensu stricto (0.803). Between cerrado sensu stricto and cerradão, the similarity value was 0.592 and between campo cerrado and cerradão, this value was 0.519 . The same pattern was also observed in Itirapina (Mantovani
1990), where campo cerrado and cerrado sensu stricto were the most similar physiognomies and the campo cerrado and cerradão, the most dissimilar ones.

Comparing the Pé-de-Gigante Reserve with cerrado sites in Campininha Farm and Emas, 76 families, 287 genera and 516 species occur in Campininha Farm (Mantovani \& Martins 1993) and 69 families, 229 genera and 358 species in Emas (Batalha et al. 1997). Despite its larger area (1269 ha), the Pé-de-Gigante Reserve presented a floristic richness similar to that found in Emas (16 ha) and lower than that found in Campininha Farm (342 ha). This could be a consequence of recent human impact on the reserve, such as timber explotation and cattle-raising, and the invasion of weeds (Pivello et al. 1999a, 1999b), but also of the prevalence of open physiognomies in Emas and Campininha Farm. Besides, differences on sampling effort could have contributed also to these results.

Table 2. Number of families, genera and species in cerrado physionomies in the Pé-de-Gigante Reserve, Santa Rita do

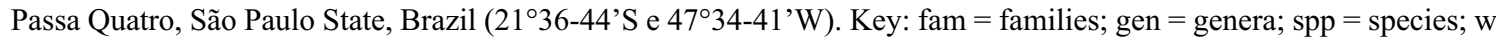
= woody species; $\mathrm{h}=$ herbaceous species; $\mathrm{w}: \mathrm{h}=$ woody to herbaceous species ratio.

\begin{tabular}{lcccccc}
\hline physiognomy & fam & gen & spp & w & h & w:h \\
\hline cerrado sensu lato & 69 & 236 & 360 & 124 & 237 & $1: 1.91$ \\
cerradão & 45 & 113 & 148 & 82 & 66 & $1: 0.80$ \\
cerrado sensu stricto & 63 & 208 & 308 & 114 & 194 & $1: 1.70$ \\
campo cerrado & 60 & 189 & 272 & 85 & 187 & $1: 2.20$ \\
\hline
\end{tabular}


The similarity index values between the Péde-Gigante Reserve and Campininha Farm and between the Pé-de-Gigante Reserve and Emas were 0.558 and 0.659 , respectively. Between Campininha Farm and Emas, this value was 0.629 (Fig. 3).

The Campininha Farm distinguished itself from the Pé-de-Gigante Reserve and from Emas by its greater floristic richness. This variation existed mainly at species level, since the genera and families remained approximately constant, as stated previously by Mantovani \& Martins (1993).

Herbaceous component - The 236 herbaceous species comprised $65.6 \%$ of the flora as a whole. The families represented only in this component accounted for $40.6 \%$ of the total number of families, a value lower than those obtained in Campininha Farm (44.7\%) (Mantovani \& Martins 1993) and Emas (49.7\%) (Batalha et al. 1997). The richest families in this component were: Asteraceae, Fabaceae, Poaceae, Rubiaceae, Bignoniaceae, Malvaceae, Apocynaceae, Euphorbiaceae, and Malpighiaceae, representing $66.5 \%$ of the total number of herbaceous species. These families are also the most representative ones in this component in other cerrado sites, such as Campininha Farm (Mantovani \& Martins 1993) and Emas (Batalha et al. 1997).

The similarity index values between the Péde-Gigante Reserve and the Campininha Farm and between the Pé-de-Gigante Reserve and Emas were 0.472 and 0.609 , respectively. Between Campininha Farm and Emas, this value was 0.580 (Fig. 3). The much higher richness found in Campininha Farm (391) contributed to separate it from Pé-de-Gigante and Emas (236 and 250 species, respectively).

There are few studies including the herbaceous species of the cerrado flora (Castro et al. 1999). The seasonality of the epigeal portion of the herbaceous species, that lasts from a few months to two years, contributes to the poor knowledge of this component. As a consequence of the short epigean cycle of many species and depending on fire frequency and intensity, the herbaceous component composition changes a lot throughout the year (Mantovani \& Martins 1993).

Woody component - The 124 woody species represented $34.4 \%$ of the whole flora. Of all sampled families, $29.0 \%$ had species only in the woody component, a value between those found in Campininha Farm (34.7\%) (Mantovani \& Martins 1993) and Emas (24.6\%) (Batalha et al. 1997).

The richest families in this component were: Fabaceae, Myrtaceae, Melastomaceae, Vochysiaceae, Malpighiaceae, Rubiaceae, Annonaceae, Bignoniaceae, and Erythroxylaceae, comprising $57.3 \%$ of collected species. These families are also the best represented in Campininha Farm (Mantovani \& Martins 1993) and Emas (Batalha et al. 1997).

In this component, 108 species were sampled in Emas and 125 in Campininha Farm. The similarity index values between the Pé-de-Gigante Reserve, Campininha Farm and Emas were all between 0.77 and 0.81 (Fig. 3). The similarity index values in this component were higher than those found in the herbaceous one, showing that the heterogeneity is higher in the herbaceous than in the woody flora. This higher heterogeneity of the herbaceous component was evident only at species level, since at genus and especially at family levels there was no pronounced difference between the two components.

Castro et al. (1999) made an extensive compilation of many floristic and phytosociological studies carried out in cerrado vegetation and elaborated a check-list of woody species. Of the species sampled here, only Myrcia guianensis (Aubl.) A. DC. (Myrtaceae) was not reported by these authors.

Andira anthelmia (Vell.) J. Macbr. (Fabaceae), Byrsonima crassa Naud. (Malpighiaceae), Anadenanthera peregrina (L.) Speg. (Mimosaceae), Eugenia langsdorffii O. Berg (Myrta- 
ceae), Myrcia pubipetala Miq. (Myrtaceae), Luehea divaricata Mart. (Tiliaceae), Syagrus romanzoffiana (Cham.) Glass. (Arecaceae), as well as Myrcia guianensis, were also not reported as ocurring in São Paulo State cerrado areas by Leitão-Filho (1992) and should be added to his list.

\section{Acknowledgements}

We are grateful to Paulo César Fernandes, for field assistance, and to FAPESP, for the financial support conceded to the first author (process 95/4290-3).

Table 1. List of species collected in cerrado physiognomies in the Pé-de-Gigante Reserve, Santa Rita do Passa Quatro,

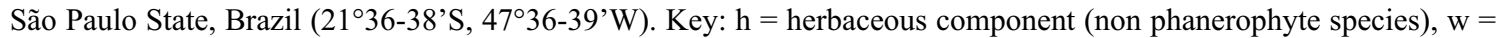
woody component (phanerophyte species); 1 = cerradão, 2 = cerrado sensu stricto, $3=$ campo cerrado; collector \# = M.A. Batalha collector number. The weedy species that does not occur spontaneously in cerrado sites, according to Mendonça et al. (1998), are marked by an asterisk.

\begin{tabular}{|c|c|c|c|c|c|}
\hline Family/ species & life-form & 1 & 2 & 3 & collector \# \\
\hline \multicolumn{6}{|l|}{ Acanthaceae } \\
\hline Hygrophila brasiliensis (Spr.) Lindau & $\mathrm{h}$ & & 2 & 3 & 1165 \\
\hline Ruellia geminiflora Kunth & $\mathrm{h}$ & 1 & 2 & & 1296 \\
\hline \multicolumn{6}{|l|}{ Alstroemeriaceae } \\
\hline Alstroemeria pulchella L. f. & $\mathrm{h}$ & & 2 & 3 & 1255 \\
\hline \multicolumn{6}{|l|}{ Amaranthaceae } \\
\hline Alternanthera brasiliana (L.) Kuntze & $\mathrm{h}$ & 1 & 2 & 3 & 724 \\
\hline Froelichia procera (Seub.) Pederson & $\mathrm{h}$ & 1 & 2 & 3 & 1523 \\
\hline Gomphrena virgata Mart. & $\mathrm{h}$ & & 2 & 3 & 762 \\
\hline Pfaffia jubata Mart. & $\mathrm{h}$ & & & 3 & 1608 \\
\hline \multicolumn{6}{|l|}{ Anacardiaceae } \\
\hline Anacardium humile A. St-Hil. & $\mathrm{h}$ & 1 & 2 & 3 & 677 \\
\hline Tapirira guianensis Aubl. & $\mathrm{w}$ & 1 & 2 & 3 & 770 \\
\hline \multicolumn{6}{|l|}{ Annonaceae } \\
\hline Annona coriacea Mart. & $\mathrm{w}$ & 1 & 2 & 3 & 671 \\
\hline Annona crassiflora Mart. & $\mathrm{w}$ & 1 & 2 & 3 & 841 \\
\hline Annona dioica A. St-Hil. & $\mathrm{h}$ & & 2 & 3 & 1509 \\
\hline Duguetia furfuracea (A. St-Hil.) Benth. \& Hook. & $\mathrm{w}$ & 1 & 2 & 3 & 1518 \\
\hline Xylopia aromatica (Lam.) Mart. & $\mathrm{w}$ & 1 & 2 & 3 & 775 \\
\hline \multicolumn{6}{|l|}{ Apiaceae } \\
\hline Didymopanax vinosum (Cham. \& Schltdl.) Seem & $\mathrm{w}$ & 1 & 2 & 3 & 611 \\
\hline Eryngium junceum Cham. \& Schltdl. & $\mathrm{h}$ & & & 3 & 854 \\
\hline \multicolumn{6}{|l|}{ Apocynaceae } \\
\hline Aspidosperma tomentosum Mart. & $\mathrm{w}$ & 1 & 2 & 3 & 1167 \\
\hline Astephanus carassensis Malme & $\mathrm{h}$ & & 2 & & 1277 \\
\hline Blepharodon nitidum (Vell.) J. Macbr. & $\mathrm{h}$ & & & 3 & 1541 \\
\hline Ditassa acerosa Mart. & $\mathrm{h}$ & & 2 & 3 & 1638 \\
\hline Ditassa obcordata Fourn. & $\mathrm{h}$ & 1 & 2 & & 1445 \\
\hline Forsteronia glabrescens Müll. Arg. & $\mathrm{h}$ & & 2 & 3 & 1506 \\
\hline Hancornia speciosa Gomez & $\mathrm{w}$ & & 2 & & 898 \\
\hline Himatanthus obovatus (Müll. Arg.) Woods. & $\mathrm{w}$ & & 2 & 3 & 1574 \\
\hline Mandevilla pohliana (Standelm.) A. Gentry & $\mathrm{h}$ & & 2 & & 987 \\
\hline Odontadenia lutea (Vell.) Markgr. & $\mathrm{h}$ & & 2 & 3 & 1347 \\
\hline Oxypetalum appendiculatum Mart. \& Zucc. & $\mathrm{h}$ & & 2 & & 692 \\
\hline Rhodocalyx rotundifolius Müll. Arg. & $\mathrm{h}$ & & 2 & & 891 \\
\hline Temnadenia violacea (Vell.) Miers & $\mathrm{h}$ & 1 & 2 & 3 & 919 \\
\hline
\end{tabular}


Table 1. (continued)

\begin{tabular}{|c|c|c|c|c|c|}
\hline Family/ species & life-form & 1 & 2 & 3 & collector \# \\
\hline \multicolumn{6}{|l|}{ Arecaceae } \\
\hline Attalea geraensis Barb. Rodr. & $\mathrm{h}$ & & 2 & 3 & 772 \\
\hline Butia paraguayensis (Barb. Rodr.) Bailey & $\mathrm{w}$ & & 2 & 3 & 818 \\
\hline Syagrus flexuosa (Mart.) Becc. & $\mathrm{w}$ & & 2 & 3 & 848 \\
\hline Syagrus loefgrenii (Mart.) Becc. & $\mathrm{h}$ & 1 & 2 & 3 & 1237 \\
\hline Syagrus romanzoffiana (Cham.) Glass. & $\mathrm{w}$ & 1 & 2 & & 1732 \\
\hline \multicolumn{6}{|l|}{ Aristolochiaceae } \\
\hline Aristolochia giberti Hook. & $\mathrm{h}$ & & & 3 & 1081 \\
\hline \multicolumn{6}{|l|}{ Asteraceae } \\
\hline Acanthospermum australe (Loefl.) Kuntze & $\mathrm{h}$ & & & 3 & 1540 \\
\hline Achyrocline satureoides (Lam.) A. DC. & $\mathrm{h}$ & & & 3 & 828 \\
\hline Aspilia reflexa Baker & $\mathrm{h}$ & & 2 & & 991 \\
\hline Baccharis dracunculifolia A. DC. & $\mathrm{h}$ & & 2 & 3 & 1433 \\
\hline Baccharis humilis Sch. Bip. & $\mathrm{h}$ & & 2 & 3 & 969 \\
\hline Baccharis rufescens Spr. & $\mathrm{h}$ & & 2 & 3 & 1703 \\
\hline Bidens gardneri Baker & $\mathrm{h}$ & 1 & 2 & 3 & 1038 \\
\hline Campuloclinium chlorolepis Baker & $\mathrm{h}$ & & 2 & 3 & 1275 \\
\hline Chaptalia integerrima (Vell.) Burk & $\mathrm{h}$ & & 2 & 3 & 1281 \\
\hline Chromolaena maximiliani (Schrad.) King \& H. Rob. & $\mathrm{h}$ & 1 & 2 & 3 & 902 \\
\hline Chromolaena squalida (A. DC.) King \& H. Rob. & $\mathrm{h}$ & & 2 & 3 & 618 \\
\hline Conyza canadensis (L.) Cronquist & $\mathrm{h}$ & & 2 & 3 & 963 \\
\hline Dasyphyllum sprengelianum (Gardner) Cabrera & $\mathrm{h}$ & 1 & 2 & & 659 \\
\hline Elephantopus biflorus Less. & $\mathrm{h}$ & & & 3 & 1263 \\
\hline Elephantopus mollis L. & $\mathrm{h}$ & 1 & 2 & 3 & 1606 \\
\hline Emilia coccinea $($ Sims) Sweet * & $\mathrm{h}$ & & 2 & 3 & 1032 \\
\hline Eremanthus erythropappus Sch. Bip. & $\mathrm{w}$ & & 2 & 3 & 1341 \\
\hline Eremanthus sphaerocephalus Baker & $\mathrm{h}$ & & 2 & 3 & 1443 \\
\hline Gochnatia barrosae Cabrera & $\mathrm{h}$ & & 2 & 3 & 1456 \\
\hline Gochnatia pulchra Cabrera & $\mathrm{h}$ & 1 & 2 & 3 & 1636 \\
\hline Kanimia oblongifolia Baker & $\mathrm{h}$ & & 2 & 3 & 750 \\
\hline Mikania cordifolia (L.) Willd. & $\mathrm{h}$ & & 2 & 3 & 1599 \\
\hline Orthopappus angustifolius (Sw.) Gleason * & $\mathrm{h}$ & & 2 & 3 & 1536 \\
\hline Piptocarpha rotundifolia (Less.) Baker & $\mathrm{w}$ & & 2 & 3 & 1025 \\
\hline Porophyllum angustissimum Gardner & $\mathrm{h}$ & & 2 & 3 & 1362 \\
\hline Porophyllum ruderale (Jacq.) Cass. * & $\mathrm{h}$ & & & 3 & 1631 \\
\hline Pterocaulon rugosum (Vahl) Malme & $\mathrm{h}$ & & & 3 & 1130 \\
\hline Stenocephalum apiculatum (A. DC.) Sch. Bip. & $\mathrm{h}$ & & 2 & & 1293 \\
\hline Tilesia baccata (A. DC.) Pruski & $\mathrm{h}$ & & 2 & 3 & 1607 \\
\hline Trichogonia salviifolia G. M. Barroso & $\mathrm{h}$ & & & 3 & 1045 \\
\hline Vernonia bardanoides Less. & $\mathrm{h}$ & 1 & 2 & 3 & 666 \\
\hline Vernonia cephalotes A. DC. & $\mathrm{h}$ & & 2 & & 1446 \\
\hline Vernonia ferruginea Less. & $\mathrm{h}$ & & 2 & & 1412 \\
\hline Vernonia herbacea (Vell.) Rusby & $\mathrm{h}$ & & & 3 & 1291 \\
\hline Vernonia holosericea Mart. & $\mathrm{h}$ & & 2 & 3 & 1352 \\
\hline Vernonia lappoides Baker & $\mathrm{h}$ & & 2 & 3 & 1173 \\
\hline Vernonia obtusata Less. & $\mathrm{h}$ & & 2 & 3 & 1375 \\
\hline Vernonia onopordioides Baker & $\mathrm{h}$ & & 2 & 3 & 1641 \\
\hline Vernonia polyanthes (Spr.) Less. & $\mathrm{h}$ & & 2 & 3 & 833 \\
\hline Vernonia rubriramea Mart. & $\mathrm{h}$ & & 2 & 3 & 1282 \\
\hline Vernonia scabra Pers. & $\mathrm{h}$ & & 2 & & 1426 \\
\hline
\end{tabular}


'Table 1. (continued)

\begin{tabular}{|c|c|c|c|c|c|}
\hline Family/ species & life-form & 1 & 2 & 3 & collector \# \\
\hline Viguiera discolor A. DC. & $\mathrm{h}$ & & 2 & 3 & 1607 \\
\hline \multicolumn{6}{|l|}{ Bignoniaceae } \\
\hline Anemopaegma arvense (Vell.) Stellfeld & $\mathrm{h}$ & & 2 & & 796 \\
\hline Anemopaegma chamberlaynii (Sims) Bur. \& K. Schum. & $\mathrm{h}$ & & & 3 & 1451 \\
\hline Arrabidaea brachypoda (A. DC.) Bur. & $\mathrm{h}$ & & 2 & 3 & 1005 \\
\hline Arrabidaea craterophora (A. DC.) Bur. & $\mathrm{h}$ & 1 & 2 & 3 & 658 \\
\hline Arrabidaea florida A. DC. & $\mathrm{h}$ & & 2 & 3 & 1615 \\
\hline Arrabidaea pulchra (Cham.) Bur. & $\mathrm{h}$ & & 2 & 3 & 1338 \\
\hline Cybistax antisyphillitica (Mart.) Mart. & $\mathrm{w}$ & & 2 & & 1175 \\
\hline Distictella mansoana (A. DC.) Urban & $\mathrm{h}$ & 1 & 2 & 3 & 964 \\
\hline Jacaranda caroba (Vell.) A. DC. & $\mathrm{h}$ & 1 & 2 & 3 & 1431 \\
\hline Jacaranda decurrens Cham. & $\mathrm{h}$ & & 2 & 3 & 751 \\
\hline Jacaranda rufa Silva Manso & $\mathrm{h}$ & & 2 & & 970 \\
\hline Memora peregrine (Miers.) Sandwith & $\mathrm{h}$ & 1 & 2 & 3 & 975 \\
\hline Pyrostegia venusta (Ker Gawl.) Bur. & $\mathrm{h}$ & 1 & 2 & 3 & 1434 \\
\hline Tabebuia aurea (Silva Manso) S. Moore. & $\mathrm{w}$ & & 2 & 3 & 1177 \\
\hline Tabebuia ochracea (Cham.) Standl. & $\mathrm{w}$ & 1 & 2 & 3 & 933 \\
\hline Zeyheria montana Mart. & $\mathrm{w}$ & & & 3 & \\
\hline \multicolumn{6}{|l|}{ Boraginaceae } \\
\hline Cordia corymbosa (L.) G. Don. & $\mathrm{h}$ & & & 3 & 1595 \\
\hline \multicolumn{6}{|l|}{ Bromeliaceae } \\
\hline Aechmea bromeliifolia (Rudge) Baker & $\mathrm{h}$ & 1 & 2 & & 878 \\
\hline Ananas ananassoides (Baker) L.B. Sm. & $\mathrm{h}$ & 1 & 2 & 3 & 737 \\
\hline Bromelia balansae Mez & $\mathrm{h}$ & 1 & 2 & 3 & 683 \\
\hline Dickia tuberosa (Vell.) Beer & $\mathrm{h}$ & & 2 & 3 & 788 \\
\hline Tillandsia geminiflora Brogn. & $\mathrm{h}$ & 1 & 2 & & 684 \\
\hline \multicolumn{6}{|l|}{ Burseraceae } \\
\hline Protium heptaphyllum (Aubl.) March & $\mathrm{w}$ & 1 & 2 & 3 & 1413 \\
\hline \multicolumn{6}{|l|}{ Cactaceae } \\
\hline Epyphyllum phyllanthus (L.) Haw. & $\mathrm{h}$ & 1 & & & 829 \\
\hline \multicolumn{6}{|l|}{ Caryocaraceae } \\
\hline Caryocar brasiliense Cambess. & $\mathrm{w}$ & 1 & 2 & 3 & 771 \\
\hline \multicolumn{6}{|l|}{ Caryophyllaceae } \\
\hline Polycarpea corymbosa (L.) Lam. & $\mathrm{h}$ & & 2 & 3 & 1511 \\
\hline \multicolumn{6}{|l|}{ Celastraceae } \\
\hline Peritassa campestris (Cambess.) A.C. Sm. & $\mathrm{w}$ & & 2 & & 1444 \\
\hline Plenckia populnea Reissek & $\mathrm{w}$ & & 2 & 3 & 949 \\
\hline Tontelea micrantha (Mart.) A.C. Sm. & $\mathrm{w}$ & & 2 & & 1097 \\
\hline \multicolumn{6}{|l|}{ Chrysobalanaceae } \\
\hline Couepia grandiflora (Mart. \& Zucc.) Hook. f. & $\mathrm{w}$ & 1 & 2 & 3 & 689 \\
\hline Licania humilis Cham. \& Schltdl. & $\mathrm{w}$ & 1 & 2 & 3 & 1358 \\
\hline Parinari excelsa Sabine & $\mathrm{h}$ & 1 & 2 & 3 & 757 \\
\hline \multicolumn{6}{|l|}{ Clusiaceae } \\
\hline Kielmeyera coriacea Mart. & $\mathrm{w}$ & & 2 & 3 & 1736 \\
\hline Kielmeyera rubriflora Cambess. & $\mathrm{w}$ & 1 & 2 & & 1270 \\
\hline Kielmeyera variabilis Mart. & $\mathrm{w}$ & 1 & 2 & 3 & 1017 \\
\hline \multicolumn{6}{|l|}{ Cochlospermaceae } \\
\hline Cochlospermum regium (Mart.) Pilg. & $\mathrm{h}$ & & 2 & 3 & 645 \\
\hline \multicolumn{6}{|l|}{ Commelinaceae } \\
\hline Commelina erecta L. & $\mathrm{h}$ & 1 & 2 & 3 & 1476 \\
\hline
\end{tabular}


Table 1. (continued)

Family/species

Connaraceae

Connarus suberosus Planch.

Rourea induta Planch.

Convolvulaceae

Ipomoea procurrens C.F.W. Meissn.

Jacquemontia tamnifolia (L.) Griseb.

Cucurbitaceae

Cayaponia espelina (Cogn.) Silva Manso

Cyperaceae

Bulbostylis hirtella (Schrad.) Urban

B.sphaerocephala (Boeck.) C.B. Clarke

Cyperus cayennensis (Lam.) Britton

Cyperus diffusus Vahl

Rhynchospora exaltata Kunth

Scleria comosa (Nees) Steud.

Dilleniaceae

Davilla elliptica A. St-Hil.

Davilla rugosa A. St-Hil.

Ebenaceae

Diospyros hispida A. DC.

Erythroxylaceae

Erythroxylum campestre A. St-Hil.

Erythroxylum pelleterianum A. St-Hil.

Erythroxylum suberosum A. St-Hil.

Erythroxylum tortuosum Mart.

Euphorbiaceae

Croton eriocladus Müll. Arg.

Croton pohlianus Müll. Arg.

Croton sclerocalyx Müll. Arg.

Manihot caerulescens Pohl

Manihot tripartita Müll. Arg.

Phyllanthus orbiculatus Müll. Arg.

Sapium glandulatum (Vell.) Pax

Sebastiania bidentata (Mart.) Pax

Sebastiania serrulata Müll. Arg.

Fabaceae

Acosmium dasycarpum (Vogel) Yakovlev

Acosmium subelegans (Mohl) Yakovlev

Aeschynomene marginata Benth.

Anadenanthera falcata (Benth.) Speg.

Anadenanthera peregrina (L.) Speg.

Andira anthelmia (Vell.) J. Macbr.

Andira laurifolia Benth.

Bauhinia rufa (Bong.) Steud.

Bowdichia virgilioides Kunth.

Centrosema venosum Mart. ex Benth.

Chamaechrista campestris Irwin \& Barneby

Chamaechrista cathartica (Mart.) Irwin \& Barneby

Chamaechrista debilis (Vogel) Irwin \& Barneby

Chamaecrista desvauxii (Collad.) Killip

\section{life-form $\quad 1 \quad 2 \quad 3 \quad$ collector \#}

\begin{tabular}{|c|c|c|c|c|}
\hline $\mathrm{w}$ & 1 & 2 & 3 & 931 \\
\hline $\mathrm{w}$ & 1 & 2 & 3 & 974 \\
\hline $\mathrm{h}$ & & 2 & 3 & 1189 \\
\hline h & & 2 & 3 & 1076 \\
\hline $\mathrm{h}$ & & 2 & 3 & 1484 \\
\hline $\mathrm{h}$ & & 2 & 3 & 1416 \\
\hline $\mathrm{h}$ & & 2 & 3 & 876 \\
\hline h & & 2 & 3 & 822 \\
\hline $\mathrm{h}$ & 1 & 2 & 3 & 1474 \\
\hline $\mathrm{h}$ & 1 & 2 & 3 & 721 \\
\hline h & 1 & 2 & 3 & 811 \\
\hline $\mathrm{w}$ & & 2 & 3 & 760 \\
\hline h & & 2 & & 993 \\
\hline $\mathrm{w}$ & 1 & 2 & 3 & 1479 \\
\hline $\mathrm{w}$ & 1 & 2 & 3 & 951 \\
\hline $\mathrm{w}$ & 1 & 2 & & 1190 \\
\hline $\mathrm{w}$ & & 2 & 3 & 1514 \\
\hline $\mathrm{w}$ & 1 & 2 & & 901 \\
\hline $\mathrm{h}$ & & 2 & 3 & 935 \\
\hline $\mathrm{h}$ & & & 3 & 917 \\
\hline $\mathrm{h}$ & & 2 & 3 & 1060 \\
\hline $\mathrm{h}$ & & 2 & & 1694 \\
\hline $\mathrm{h}$ & & 2 & 3 & 918 \\
\hline $\mathrm{h}$ & & 2 & 3 & 1359 \\
\hline $\mathrm{h}$ & & & 3 & 1061 \\
\hline h & & & 3 & 1034 \\
\hline h & & 2 & 3 & 989 \\
\hline $\mathrm{w}$ & 1 & & & 1297 \\
\hline $\mathrm{w}$ & & 2 & & 915 \\
\hline $\mathrm{h}$ & & 2 & 3 & 1327 \\
\hline $\mathrm{w}$ & 1 & 2 & 3 & 1589 \\
\hline $\mathrm{w}$ & & 2 & & 1082 \\
\hline $\mathrm{w}$ & & 2 & & 1033 \\
\hline $\mathrm{h}$ & & 2 & 3 & 699 \\
\hline $\mathrm{w}$ & 1 & 2 & 3 & 1000 \\
\hline w & 1 & 2 & & 1071 \\
\hline $\mathrm{h}$ & & & 3 & 1199 \\
\hline $\mathrm{h}$ & & 2 & 3 & 948 \\
\hline $\mathrm{h}$ & & & 3 & 1543 \\
\hline $\mathrm{h}$ & & & 3 & 954 \\
\hline $\mathrm{h}$ & & 2 & 3 & 992 \\
\hline
\end{tabular}


Table 1. (continued)

\begin{tabular}{|c|c|c|c|c|c|}
\hline Family/ species & life-form & 1 & 2 & 3 & collector \# \\
\hline Chamaecrista flexuosa (L.) Greene & $\mathrm{h}$ & & 2 & 3 & 639 \\
\hline Chamaecrista rotundifolia (Pers.) Greene & $\mathrm{h}$ & & & 3 & 1332 \\
\hline Clitoria falcata Lam. & $\mathrm{h}$ & & & 3 & 1612 \\
\hline Clitoria laurifolia Poir. & $\mathrm{h}$ & & & 3 & 1480 \\
\hline Copaifera langsdorffii Desf. & $\mathrm{w}$ & 1 & 2 & 3 & 642 \\
\hline Crotalaria vitellina Ker Gawl. & $\mathrm{h}$ & & 2 & 3 & 1009 \\
\hline Dalbergia miscolobium Benth. & $\mathrm{w}$ & 1 & 2 & 3 & 1344 \\
\hline Deguelia nitidula (Benth.) Az.-Tozzi & $\mathrm{h}$ & & 2 & & 1743 \\
\hline Dimorphandra mollis Benth. & $\mathrm{w}$ & 1 & 2 & 3 & 1706 \\
\hline Dyptichandra aurantiaca Tul. & $\mathrm{w}$ & 1 & 2 & 3 & 1181 \\
\hline Enterolobium gummiferum (Mart.) Macbr. & $\mathrm{W}$ & & 2 & & 1470 \\
\hline Eriosema crinitum (Kunth) Gardner & $\mathrm{h}$ & & 2 & 3 & 1298 \\
\hline Galactia decumbens (Benth.) Hassl. & $\mathrm{h}$ & & & 3 & 960 \\
\hline Galactia grewiifolia (Benth.) Taub. & $\mathrm{h}$ & 1 & 2 & 3 & 1065 \\
\hline Hymenaea stigonocarpa Mart. & $\mathrm{w}$ & 1 & 2 & 3 & 1027 \\
\hline Indigofera suffruticosa Mill. & $\mathrm{h}$ & & 2 & & 1414 \\
\hline Machaerium acutifolium Vogel & $\mathrm{w}$ & 1 & 2 & 3 & 1060 \\
\hline Macroptilium gracile (Poepp. ex Benth.) Urban & $\mathrm{h}$ & & 2 & & 1357 \\
\hline Mimosa debilis Humb. \& Bonpl. ex Willd. & $\mathrm{h}$ & & & 3 & 1616 \\
\hline Mimosa gracilis Benth. & $\mathrm{h}$ & & 2 & 3 & 1016 \\
\hline Mimosa pigra L. * & $\mathrm{h}$ & & 2 & & 1010 \\
\hline Mimosa xanthocentra Mart. & $\mathrm{h}$ & & 2 & 3 & 1584 \\
\hline Periandra mediterranea (Vell.) Taub. & $\mathrm{h}$ & 1 & 2 & 3 & 1268 \\
\hline Plathymenia reticulata Benth. & $\mathrm{w}$ & 1 & 2 & & 1521 \\
\hline Platypodium elegans Vogel & $\mathrm{W}$ & 1 & & & 1388 \\
\hline Pterodon pubescens Benth. & $\mathrm{w}$ & 1 & 2 & 3 & 661 \\
\hline Rhynchosia melanocarpa Grear & $\mathrm{h}$ & & 2 & 3 & 1635 \\
\hline Sclerolobium paniculatum Vogel & $\mathrm{W}$ & 1 & 2 & & 1252 \\
\hline Senna rugosa (G. Don.) Irwin \& Barneby & $\mathrm{w}$ & & 2 & 3 & 693 \\
\hline Senna silvestris (Vell.) Irwin \& Barneby & $\mathrm{w}$ & 1 & 2 & 0 & 1184 \\
\hline Stryphnodendron adstringens (Mart.) Coville & $\mathrm{w}$ & & 2 & 3 & 1711 \\
\hline Stryphnodendron obovatum Mart. & $\mathrm{w}$ & 1 & 2 & & 1048 \\
\hline Stylosanthes gracilis Kunth & $\mathrm{h}$ & & 2 & 3 & 1308 \\
\hline Stylosanthes guianensis (Aubl.) Sw. & $\mathrm{h}$ & & 2 & & 1497 \\
\hline Vatairea macrocarpa (Benth.) Ducke & $\mathrm{w}$ & 1 & 2 & 3 & 1200 \\
\hline Zornia latifolia $\mathrm{Sm}$. & $\mathrm{h}$ & & 2 & 3 & 1491 \\
\hline \multicolumn{6}{|l|}{ Flacourtiaceae } \\
\hline Casearia grandiflora Cambess. & $\mathrm{w}$ & 1 & 2 & 0 & 1023 \\
\hline Casearia sylvestris $\mathrm{Sw}$. & $\mathrm{w}$ & 1 & 2 & 3 & 672 \\
\hline \multicolumn{6}{|l|}{ Iridaceae } \\
\hline Trimezia juncifolia (K1.) Kunth & $\mathrm{h}$ & 1 & 2 & 3 & 862 \\
\hline \multicolumn{6}{|l|}{ Lamiaceae } \\
\hline Hyptidodendron canum (Pohl ex Benth.) Harley & $\mathrm{w}$ & 1 & 2 & 3 & 657 \\
\hline Hyptis brevipes Poit. * & $\mathrm{h}$ & 1 & 2 & 3 & 1335 \\
\hline Hyptis eriophylla Pohl & $\mathrm{h}$ & & 2 & 3 & 1640 \\
\hline Hyptis mutabilis (A. Rich.) Briq. & $\mathrm{h}$ & & 2 & & 1316 \\
\hline Hyptis reticulata Mart. & $\mathrm{h}$ & & 2 & 3 & 747 \\
\hline Hyptis rugosa Benth. & $\mathrm{h}$ & & 2 & 3 & 1356 \\
\hline Peltodon tomentosus Pohl & $\mathrm{h}$ & & 2 & 3 & 1534 \\
\hline
\end{tabular}


Table 1. (continued)

\begin{tabular}{|c|c|c|c|c|c|}
\hline Family/ species & life-form & 1 & 2 & 3 & collector \# \\
\hline Cassytha americana Nees & $\mathrm{h}$ & & 2 & & 1462 \\
\hline Ocotea corymbosa (Meiss.) Mez & $\mathrm{w}$ & 1 & 2 & 3 & 600 \\
\hline Ocotea pulchella Mart. & $\mathrm{w}$ & & 2 & 3 & 1038 \\
\hline \multicolumn{6}{|l|}{ Loganiaceae } \\
\hline Strychnos bicolor Progel & $\mathrm{h}$ & 1 & 2 & & 1585 \\
\hline Strychnos pseudoquina A. St-Hil. & $\mathrm{w}$ & 1 & & & 743 \\
\hline \multicolumn{6}{|l|}{ Loranthaceae } \\
\hline Psittacanthus robustus Mart. & $\mathrm{h}$ & & 2 & & 710 \\
\hline \multicolumn{6}{|l|}{ Lythraceae } \\
\hline Cuphea carthaginensis (Jacq.) Macbr. & $\mathrm{h}$ & & 2 & 3 & 1561 \\
\hline Lafoensia pacari A. St-Hil. & $\mathrm{w}$ & & 2 & 3 & 1264 \\
\hline \multicolumn{6}{|l|}{ Malpighiaceae } \\
\hline Banisteriopsis argyrophylla (A. Juss.) B. Gates & $\mathrm{h}$ & 1 & & & 1287 \\
\hline Banisteriopsis campestris (A. Juss.) Little & $\mathrm{h}$ & & 2 & 3 & 1210 \\
\hline Banisteriopsis laevifolia (A. Juss.) B. Gates & $\mathrm{h}$ & & 2 & 3 & 1560 \\
\hline Banisteriopsis pubipetala (A. Juss.) Cuatrec. & $\mathrm{h}$ & 1 & 2 & 3 & 628 \\
\hline Banisteriopsis stellaris (Griseb.) B. Gates & $\mathrm{h}$ & 1 & 2 & 3 & 1024 \\
\hline Banisteriopsis variabilis B. Gates & $\mathrm{h}$ & 1 & 2 & 3 & 784 \\
\hline Byrsonima coccolobifolia A. Juss. & $\mathrm{w}$ & 1 & 2 & 3 & 1036 \\
\hline Byrsonima crassa Nied. & $\mathrm{w}$ & & 2 & & 1019 \\
\hline Byrsonima intermedia A. Juss. & $\mathrm{w}$ & & 2 & 3 & 606 \\
\hline Byrsonima verbascifolia (L.) Rich ex A. Juss. & $\mathrm{w}$ & & 2 & 3 & 1354 \\
\hline Heteropteris byrsonimifolia A. Juss. & $\mathrm{w}$ & & 2 & 3 & 1621 \\
\hline Heteropteris umbellata A. Juss. & $\mathrm{h}$ & & 2 & 3 & 853 \\
\hline Mascagnia cordifolia (A. Juss.) Griseb. & $\mathrm{h}$ & 1 & 2 & 3 & 1494 \\
\hline Peixotoa tomentosa A. Juss. & $\mathrm{h}$ & & 2 & 3 & 1610 \\
\hline \multicolumn{6}{|l|}{ Malvaceae } \\
\hline Byttneria sagittifolia A. St-Hil. & $\mathrm{h}$ & 1 & 2 & 3 & 1493 \\
\hline Eriotheca gracilipes (K. Schum.) A. Robyns & $\mathrm{w}$ & 1 & 2 & 3 & 1427 \\
\hline Helicteres sacarolha A. St-Hil. & $\mathrm{h}$ & & & 3 & 1235 \\
\hline Luehea divaricata Mart. & $\mathrm{w}$ & 1 & 2 & 3 & 582 \\
\hline Pavonia communis A. St-Hil. & $\mathrm{h}$ & & & 3 & 1038 \\
\hline Pavonia hexaphylla (S. Moore) Krapov. & $\mathrm{h}$ & & 2 & 3 & 1024 \\
\hline Peltaea edouardii (Hochr.) Krapov. \& Cristóbal & $\mathrm{h}$ & & & 3 & 1056 \\
\hline Pseudobombax longiflorum (Mart. \& Zucc.) A. Robyns & $\mathrm{w}$ & 1 & & & 1288 \\
\hline Sida glaziovii K. Schum. * & $\mathrm{h}$ & & & 3 & 634 \\
\hline Sida linifolia A. Juss. * & $\mathrm{h}$ & & 2 & 3 & 1624 \\
\hline Sida rhombifolia L. * & $\mathrm{h}$ & & & 3 & 1051 \\
\hline Sida urens L. * & $\mathrm{h}$ & & & 3 & 1644 \\
\hline Waltheria americana $\mathrm{L}$. & $\mathrm{h}$ & & 2 & 3 & 835 \\
\hline Waltheria communis L. & $\mathrm{h}$ & & 2 & 3 & 984 \\
\hline \multicolumn{6}{|l|}{ Melastomataceae } \\
\hline Leandra lacunosa Cogn. & $\mathrm{w}$ & & 2 & 3 & 620 \\
\hline Miconia albicans Triana & $\mathrm{w}$ & 1 & 2 & 3 & 1629 \\
\hline Miconia fallax A. DC. & $\mathrm{h}$ & 1 & 2 & 3 & 1575 \\
\hline Miconia ligustroides Naudin & $\mathrm{w}$ & 1 & 2 & 3 & 1639 \\
\hline Miconia rubiginosa (Bonpl.) A. DC. & $\mathrm{w}$ & 1 & 2 & 3 & 1522 \\
\hline Miconia stenostachya A. DC. & $\mathrm{w}$ & 1 & 2 & 3 & 732 \\
\hline Tibouchina stenocarpa (A. DC.) Cogn. & $\mathrm{w}$ & 1 & 2 & 3 & 662 \\
\hline
\end{tabular}


Table 1. (continued)

\begin{tabular}{|c|c|c|c|c|c|}
\hline Family/ species & life-form & 1 & 2 & 3 & collector \# \\
\hline Cissampelos ovalifolia Ruiz \& Pav. & $\mathrm{h}$ & & 2 & 3 & 1556 \\
\hline \multicolumn{6}{|l|}{ Monimiaceae } \\
\hline Siparuna guianensis Aubl. & $\mathrm{w}$ & & 2 & & 1495 \\
\hline \multicolumn{6}{|l|}{ Moraceae } \\
\hline Brosimum gaudichaudii Trècul & $\mathrm{w}$ & & 2 & & 820 \\
\hline Ficus citrifolia Mill. & $\mathrm{w}$ & & 2 & & 1011 \\
\hline \multicolumn{6}{|l|}{ Myristicaceae } \\
\hline Virola sebifera Aubl. & $\mathrm{w}$ & 1 & 2 & & 1576 \\
\hline \multicolumn{6}{|l|}{ Myrtaceae } \\
\hline Campomanesia pubescens (A. DC.) O. Berg & $\mathrm{w}$ & & 2 & 3 & 1050 \\
\hline Eugenia aurata $\mathrm{O}$. Berg & $\mathrm{w}$ & & 2 & 3 & 1634 \\
\hline Eugenia bimarginata A. DC. & $\mathrm{w}$ & 1 & 2 & 3 & 1455 \\
\hline Eugenia hiemalis Cambess. & $\mathrm{w}$ & & 2 & & 1314 \\
\hline Eugenia langsdorffii O. Berg & $\mathrm{w}$ & 1 & 2 & 3 & 1475 \\
\hline Eugenia livida $\mathrm{O}$. Berg & $\mathrm{w}$ & 1 & 2 & 3 & 1389 \\
\hline Eugenia punicifolia (Kunth) A. DC. & $\mathrm{w}$ & 1 & 2 & 3 & 1222 \\
\hline Myrcia bella Cambess. & $\mathrm{w}$ & 1 & 2 & 3 & 965 \\
\hline Myrcia guianensis (Aubl.) A. DC. & $\mathrm{w}$ & & 2 & & 1220 \\
\hline Myrcia lasiantha A. DC. & $\mathrm{w}$ & 1 & 2 & 3 & 754 \\
\hline Myrcia pubipetala Miq. & $\mathrm{w}$ & 1 & & & 1215 \\
\hline Myrcia tomentosa (Aubl.) A. DC. & $\mathrm{w}$ & 1 & 2 & & 940 \\
\hline Myrcia uberavensis O. Berg & $\mathrm{w}$ & 1 & 2 & 3 & 921 \\
\hline Psidium australe Cambess. & $\mathrm{h}$ & & 2 & 3 & 947 \\
\hline Psidium cinereum Mart. & $\mathrm{h}$ & & 2 & 3 & 877 \\
\hline \multicolumn{6}{|l|}{ Nyctaginaceae } \\
\hline Guapira noxia (Netto) Lund & $\mathrm{w}$ & 1 & 2 & & 794 \\
\hline Neea theifera Oerst. & $\mathrm{w}$ & & 2 & 3 & 1054 \\
\hline \multicolumn{6}{|l|}{ Ochnaceae } \\
\hline Ouratea castaneaefolia (A. DC.) Engl. & $\mathrm{w}$ & 1 & & & 1007 \\
\hline Ouratea spectabilis (Mart.) Engl. & $\mathrm{w}$ & 1 & 2 & 3 & 909 \\
\hline \multicolumn{6}{|l|}{ Orchidaceae } \\
\hline Galeandra montana Barb. Rodr. & $\mathrm{h}$ & & 2 & & 1096 \\
\hline Ionopsis paniculata Lindl. & $\mathrm{h}$ & 1 & & & 1460 \\
\hline \multicolumn{6}{|l|}{ Oxalidaceae } \\
\hline Oxalis physocallyx Zucc. & $\mathrm{h}$ & & 2 & 3 & 1517 \\
\hline \multicolumn{6}{|l|}{ Poaceae } \\
\hline Andropogon leucostachys Kunth & $\mathrm{h}$ & & 2 & 3 & 980 \\
\hline Aristida jubata (Arechav.) Herter & $\mathrm{h}$ & & 2 & 3 & 612 \\
\hline Axonopus barbigerus (Kunth) Hitchc. & $\mathrm{h}$ & & 2 & 3 & 1577 \\
\hline Axonopus marginatus (Trin.) Chase & $\mathrm{h}$ & & 2 & 3 & 968 \\
\hline Brachiaria decumbens Stapf* & $\mathrm{h}$ & & 2 & 3 & 851 \\
\hline Chloris barbata (L.) Sw. & $\mathrm{h}$ & & 2 & & 1563 \\
\hline Digitaria insularis (L.) Fedde & $\mathrm{h}$ & 1 & 2 & 3 & 857 \\
\hline Echinolaena inflexa (Poir.) Chase & $\mathrm{h}$ & 1 & 2 & 3 & 1698 \\
\hline Eragrostis airoides Ness & $\mathrm{h}$ & & 2 & 3 & 1058 \\
\hline Eragrostis articulata (Schrank) Nees & $\mathrm{h}$ & & 2 & & 1579 \\
\hline Eragrostis maypurensis (Kunth) Steud. & $\mathrm{h}$ & & 2 & 3 & 1379 \\
\hline Gymnopogon foliosus (Willd.) Nees & $\mathrm{h}$ & & 2 & 3 & 1325 \\
\hline Ichnanthus sericeus Hack. & $\mathrm{h}$ & 1 & 2 & 3 & 846 \\
\hline Loudetiopsis chrysothrix (Nees) Conert & $\mathrm{h}$ & 1 & 2 & 3 & 1067 \\
\hline
\end{tabular}


Table 1. (continued)

\begin{tabular}{|c|c|c|c|c|c|}
\hline Family/ species & life-form & 1 & 2 & 3 & collector \# \\
\hline Melinis minutiflora P. Beauv. * & $\mathrm{h}$ & 1 & 2 & 3 & 622 \\
\hline Panicum cayennensis Lam. & $\mathrm{h}$ & & 2 & 3 & 1331 \\
\hline Panicum maximum Jacq. * & $\mathrm{h}$ & 1 & 2 & & 1266 \\
\hline Panicum olyroides Kunth & $\mathrm{h}$ & & 2 & 3 & 832 \\
\hline Panicum parvifolium Lam. & $\mathrm{h}$ & & 2 & 3 & 1244 \\
\hline Panicum procurrens Nees & $\mathrm{h}$ & & 2 & & 1722 \\
\hline Panicum repens L. * & $\mathrm{h}$ & & 2 & & 1477 \\
\hline Rhynchelitrum repens (Nees) C.E. Hubb. * & $\mathrm{h}$ & & 2 & 3 & 844 \\
\hline Schyzachirium condensatum (Kunth) Nees & $\mathrm{h}$ & & 2 & 3 & 1442 \\
\hline Setaria geniculata (L.) P. Beauv. * & $\mathrm{h}$ & & 2 & & 1350 \\
\hline Sporolobus indicus (L.) R. Br. & $\mathrm{h}$ & & & 3 & 1246 \\
\hline Tristachya leiostachya Nees & $\mathrm{h}$ & & 2 & 3 & 1098 \\
\hline \multicolumn{6}{|l|}{ Polygalaceae } \\
\hline Bredemeyera floribunda Willd. & $\mathrm{w}$ & 1 & 2 & 3 & 1226 \\
\hline Securidaca tomentosa A. St-Hil. & $\mathrm{h}$ & & 2 & & 1001 \\
\hline \multicolumn{6}{|l|}{ Polypodiaceae } \\
\hline Adiantum fructuosum $\mathrm{Spr}$. & $\mathrm{h}$ & 1 & 2 & 3 & 660 \\
\hline Polypodium latipes Langsd. \& Fisch. & $\mathrm{h}$ & 1 & 2 & 3 & 1013 \\
\hline \multicolumn{6}{|l|}{ Portulacaceae } \\
\hline Portulaca hirsutissima Cambess. & $\mathrm{h}$ & & 2 & 3 & 836 \\
\hline Portulaca mucronata Link & $\mathrm{h}$ & & 2 & 3 & 1014 \\
\hline Tallinum paniculatum (Jacq.) Gaertn. & $\mathrm{h}$ & 1 & 2 & & 999 \\
\hline \multicolumn{6}{|l|}{ Proteaceae } \\
\hline Roupala montana Aubl. & $\mathrm{w}$ & 1 & 2 & 3 & 663 \\
\hline \multicolumn{6}{|l|}{ Rhamnaceae } \\
\hline Crumenaria polygaloides Reissek & $\mathrm{h}$ & & 2 & & 679 \\
\hline \multicolumn{6}{|l|}{ Rosaceae } \\
\hline Prunus sellowii $\mathrm{Sm}$. & $\mathrm{w}$ & & 2 & & 986 \\
\hline \multicolumn{6}{|l|}{ Rubiaceae } \\
\hline Alibertia macrophylla K. Schum. & $\mathrm{w}$ & 1 & 2 & & 1087 \\
\hline Alibertia sessilis (Vell.) K. Schum. & $\mathrm{h}$ & 1 & 2 & 3 & 1360 \\
\hline Amaioua guianensis Aubl. & $\mathrm{w}$ & 1 & & & 985 \\
\hline Borreria verticilata (L.) Mey. & $\mathrm{h}$ & 1 & 2 & 3 & 1569 \\
\hline Borreria warmingii K. Schum. & $\mathrm{h}$ & & 2 & 3 & 941 \\
\hline Coccocypselum lanceolatum (Ruiz \& Pav.) Pers. & $\mathrm{h}$ & 1 & 2 & & 1261 \\
\hline Declieuxia fruticosa (Roem. \& Schult) Kuntze & $\mathrm{h}$ & 1 & 2 & 3 & 1087 \\
\hline Diodia schumanii Standl. ex Bacigalupo & $\mathrm{h}$ & 1 & 2 & 3 & 1090 \\
\hline Diodia teres Walt. & $\mathrm{h}$ & & 2 & & 1366 \\
\hline Palicourea coriacea (Cham.) K. Schum. & $\mathrm{h}$ & 1 & 2 & 3 & 1526 \\
\hline Palicourea rigida Kunth & $\mathrm{w}$ & 1 & 2 & 3 & 983 \\
\hline Psychotria barbiflora A. DC. & $\mathrm{h}$ & 1 & & & 1306 \\
\hline Psychotria capitata Ruiz \& Pav. & $\mathrm{h}$ & 1 & & & 1468 \\
\hline Psychotria deflexa A. DC. & $\mathrm{h}$ & & 2 & & 971 \\
\hline Psychotria tricholoba Müll. Arg. & $\mathrm{h}$ & 1 & 2 & & 972 \\
\hline Rudgea viburnoides (Cham.) Benth. & $\mathrm{w}$ & 1 & & & 1093 \\
\hline Sabicea brasiliensis Wernham & $\mathrm{h}$ & & & 3 & 967 \\
\hline Tocoyena formosa (Cham. \& Schltdl.) K. Schum. & $\mathrm{w}$ & & 2 & 3 & 1515 \\
\hline \multicolumn{6}{|l|}{ Sapindaceae } \\
\hline Magonia pubescens A. St-Hil. & $\mathrm{w}$ & & 2 & & 1250 \\
\hline Matayba elaeagnoides Radlk. & $\mathrm{w}$ & & 2 & 3 & 1679 \\
\hline
\end{tabular}


Table 1. (continued)

\begin{tabular}{|c|c|c|c|c|c|}
\hline Family/species & life-form & 1 & 2 & 3 & collector \# \\
\hline Paullinia elegans Cambess. & $\mathrm{h}$ & 1 & 2 & 3 & 864 \\
\hline Serjania erecta Radlk. & $\mathrm{h}$ & 1 & 2 & 3 & 1554 \\
\hline Serjania lethalis A. St-Hil. & $\mathrm{h}$ & 1 & 2 & 3 & 664 \\
\hline Serjania reticulata Cambess. & $\mathrm{h}$ & 1 & 2 & 3 & 1329 \\
\hline Talisia angustifolia Raddi & $\mathrm{h}$ & 1 & 2 & 3 & 914 \\
\hline Toulicia tomentosa Radlk. & $\mathrm{w}$ & & 2 & 3 & 693 \\
\hline \multicolumn{6}{|l|}{ Sapotaceae } \\
\hline Pouteria ramiflora (Mart.) Radlk. & $\mathrm{w}$ & 1 & 2 & 3 & 1524 \\
\hline Pouteria subcaerulea Pierre ex Dubard & $\mathrm{h}$ & 1 & 2 & 3 & 655 \\
\hline Pouteria torta (Mart.) Radlk. & $\mathrm{w}$ & 1 & 2 & 3 & 1428 \\
\hline Pradosia brevipes (Pierre) Penn. & $\mathrm{h}$ & 1 & 2 & 3 & 887 \\
\hline \multicolumn{6}{|l|}{ Schizaeaceae } \\
\hline Anemia ferruginea Kunth & $\mathrm{h}$ & & 2 & 3 & 1260 \\
\hline \multicolumn{6}{|l|}{ Scrophulariaceae } \\
\hline Buchnera lavandulacea Cham. \& Schltdl. & $\mathrm{h}$ & & & 3 & 1292 \\
\hline \multicolumn{6}{|l|}{ Smilacaceae } \\
\hline \multirow{2}{*}{\multicolumn{6}{|c|}{ Solanaceae }} \\
\hline & & & & & \\
\hline Solanum erianthum D. Don * & $\mathrm{w}$ & & 2 & 3 & 868 \\
\hline Solanum lycocarpum A. St-Hil. & $\mathrm{h}$ & & 2 & 3 & 929 \\
\hline Solanum palinacanthum Dunal & $\mathrm{h}$ & & 2 & 3 & 656 \\
\hline \multicolumn{6}{|l|}{ Styracaceae } \\
\hline Styrax ferrugineus Nees \& Mart. & $\mathrm{w}$ & 1 & 2 & 3 & 1324 \\
\hline \multicolumn{6}{|l|}{ Turneraceae } \\
\hline Piriqueta rosea (Cambess.) Urban & $\mathrm{h}$ & & & 3 & 1055 \\
\hline \multicolumn{6}{|l|}{ Verbenaceae } \\
\hline Aegiphila lhotzkiana Cham. & $\mathrm{w}$ & & 2 & & 906 \\
\hline Lantana camara $\mathrm{L}$. & $\mathrm{h}$ & 1 & 2 & 3 & 1597 \\
\hline Lantana hypoleuca Briq. & $\mathrm{h}$ & 1 & 2 & 3 & 939 \\
\hline Lippia lasiocalycina Cham. & $\mathrm{h}$ & & 2 & & 821 \\
\hline Lippia lupulina Cham. & $\mathrm{h}$ & & 2 & & 870 \\
\hline Lippia salviifolia Cham. & $\mathrm{h}$ & 1 & 2 & 3 & 911 \\
\hline Stachytarpheta maximilliani Schauer & $\mathrm{h}$ & & & 3 & 1548 \\
\hline \multicolumn{6}{|l|}{ Vitaceae } \\
\hline Cissus erosa Rich. & $\mathrm{h}$ & & 2 & 3 & 953 \\
\hline Cissus inundata (Baker) Planch. & $\mathrm{h}$ & & 2 & 3 & 826 \\
\hline \multicolumn{6}{|l|}{ Vochysiaceae } \\
\hline Qualea dichotoma Warm. & $\mathrm{w}$ & 1 & 2 & 3 & 729 \\
\hline Qualea grandiflora Mart. & $\mathrm{w}$ & 1 & 2 & 3 & 650 \\
\hline Qualea multiflora Mart. & $\mathrm{w}$ & 1 & 2 & & 981 \\
\hline Qualea parviflora Mart. & $\mathrm{w}$ & 1 & 2 & 3 & 676 \\
\hline Vochysia cinamommea Pohl & $\mathrm{w}$ & 1 & 2 & 3 & 892 \\
\hline Vochysia tucanorum Mart. & $\mathrm{w}$ & 1 & 2 & 3 & 819 \\
\hline
\end{tabular}




\section{References}

Batalha, M.A., Aragaki, S. \& Mantovani, W. 1997. Florística do cerrado em Emas (Pirassununga, SP). Boletim de Botânica da Universidade de São Paulo $16: 49-64$.

Bitencourt, M.D.; Mesquita Jr., H. N. de; Mantovani, W.; Batalha, M.A. \& Pivello, V.R. 1997. Identificação de fisionomias de cerrado com imagem índice de vegetação pp. 316-320. In L.L. Leite \& C.H. Saito (eds.). Contribuição ao conhecimento ecológico do cerrado. Universidade de Brasília, Brasília.

Castro, A.A.J.F.; Martins, F.R.; Tamashiro, J.Y. \& Shepherd, G.J. 1999. How rich is the flora of Brazilian cerrados? Annals of Missouri Botanical Garden 86:192-224.

Coutinho, L.M. 1978. O conceito do cerrado. Revista Brasileira de Botânica 1:17-23.

Goodland, R. 1979. Análise ecológica da vegetação de cerrado pp. 61-186. In R. Goodland \& M.G. Ferri (eds.). Ecologia do cerrado. Itatiaia, Belo Horizonte.

Jongman, R.H.G.; Ter Braak, C.J.F. \& van Tongeren, O.F.R. 1995. Data analysis in community and landscape ecology. Cambridge University, Cambridge.

Judd, W.S.; Campbell, C.S.; Kellog, E.A. \& Stevens, P.F. 1999. Plant systematics: a phylogenetic approach. Sinauer, Sunderland.

Köppen, W. 1948. Climatologia. Fondo de Cultura Económica, México.

Leitão Filho, H. de F. 1992. A flora arbórea dos cerrados do estado de São Paulo. Hoehnea 19:121-163.

Magurran, A.E. 1988. Ecological diversity and its measurement. Princeton University, Princeton.

Mantovani, W. 1990. Variação da flora arbustivo-arbórea de diversas fisionomias de cerrado, em Itirapina, estado de São Paulo pp. 125-135. In Anais do XLI Congresso Nacional de Botânica, Curitiba 1990. Sociedade Botânica do Brasil, Curitiba.

Mantovani, W. \& Martins, F.R. 1993. Florística do cerrado na reserva biológica de Moji Guaçu, SP. Acta Botanica Brasilica 7:33-60.

Mendonça, R.C. de; Felfili, J.M.; Walter, B.M.T.; Silva Júnior, M.C. da; Rezende, A.V.; Filgueiras, T.S. \& Nogueira, P.E. 1998. A flora vascular do cerrado pp. 279-556. In S.M. Sano \& S.P. de Almeida (eds.). Cerrado - ambiente e flora. EMBRAPA, Planaltina.

Mueller-Dombois, D. \& Ellenberg, H. 1974. Aims and methods of vegetation ecology. John Wiley \& Sons, New York.
Pivello, V.R.; Bitencourt, M.D.; Mantovani, W.; Mesquita Jr., H.N. de; Batalha, M.A. \& Shida, C. 1998. Proposta de zoneamento ecológico para a reserva de cerrado Pé-de-Gigante (Santa Rita do Passa Quatro, SP). Brazilian Journal of Ecology 2:108-118.

Pivello, V.R.; Bitencourt, M.D.; Mesquita Jr., H.N. de \& Batalha, M.A. 1999a. Banco de dados em SIG para ecologia aplicada: exemplo do cerrado Pé-deGigante, S.P. Caderno de Informações Georreferenciadas 1:http://www.cpa.unicamp.br/ revista/cigvln3a4.html.

Pivello, V.R.; Carvalho, V.M.C.; Lopes, P.F.; Peccinini, A.A. \& Rosso, S. 1999b. Abundance and distribution of native and alien grasses in a "cerrado" (Brazilian savanna) Biological Reserve. Biotropica 31:71-82.

Pivello, V.R.; Shida, C.N. \& Meirelles, S.T. 1999c. Alien grasses in Brazilian savannas: a threat to the biodiversity. Biodiversity and Conservation 8:1281-1294.

Ratter, J.A. 1980. Notes on the vegetation of Fazenda Água Limpa (Brasília - DF, Brasil). Royal Botanical Garden, Edinburgh.

Ratter, J.A., Ribeiro, J.F. \& Bridgewater, S. 1992. The Brazilian cerrado vegetation and threats to its biodiversity. Annals of Botany 80:223-230.

Thornthwaite, C.W. \& Mather, J.R. 1955. The water balance. Centerton, New Jersey.

Warming, E. 1892. Lagoa Santa, contribuição para a geografia fitobiológica pp. 1-284. In E. Warming \& M.G. Ferri (eds.). 1973. Lagoa Santa e a vegetação dos cerrados brasileiros. Itatiaia, Belo Horizonte. 